\title{
From Bouveret's Syndrome to Gallstone Ileus: The Journey of a Migrating Stone!
}

\author{
Fady G. Haddad ${ }^{1}$, Wissam Mansour ${ }^{2}$, Joseph Mansour ${ }^{3}$, Liliane Deeb ${ }^{1}$ \\ 1. Department of Gastroenterology and Hepatology, Staten Island University Hospital, New York 2. \\ Department of Pulmonary Critical Care, Staten Island University Hospital, Northwell Health 3. \\ Department of Radiology, Saint Louis University
}

$\square$ Corresponding author: Fady G. Haddad, fadi.haddad7@hotmail.com Disclosures can be found in Additional Information at the end of the article

\section{Abstract}

Bouveret's syndrome, first described in 1896, is an unusual cause of gastric outlet obstruction secondary to large gallstone impaction in the proximal duodenum after migration through a cholecystoduodenal fistula. Stone migration has been previously described after endoscopic or surgical fragmentation. However, this is the first reported case, in our knowledge, where the stone migrated after oral contrast administration a few days after the onset of symptoms, causing a distal gallstone ileus.

Categories: Internal Medicine, Gastroenterology, General Surgery

Keywords: bouveret syndrome, gallstones, gall stone ileus

\section{Introduction}

Bouveret's syndrome, first described in 1896, is an unusual cause of gastric outlet obstruction secondary to large gallstone impaction in the proximal duodenum after migration through a cholecystoduodenal fistula. Stone migration to the distal small intestine has been described after primary endoscopic or surgical fragmentation of the original impacted gallstone [1]. Our case report is the first, in our knowledge, to describe the migration of the duodenal stone after the oral administration of water-soluble hyperosmolar contrast agent, used while performing abdominal computed tomography (CT) imaging. This phenomenon occurred 48 hours after contrast intake and caused a distal jejunal gallstone ileus.

\section{Case Presentation}

A 61-year old female with diabetes mellitus and hypertension presented with a five-day history of epigastric pain and postprandial vomiting. A physical examination revealed a soft abdomen with mild epigastric tenderness. Labs were significant for an acute kidney injury. An abdominal CT scan following water-soluble hyperosmolar radiocontrast administration showed a $3.5 \mathrm{~cm}$ intraluminal calculus at the level of the third duodenal segment, diffuse pneumobilia, and an air-containing gallbladder, suggesting Bouveret's syndrome (Figure 1).

Haddad et al. This is an open access article distributed under the terms of the Creative Commons Attribution License CC-BY 3.0., which permits unrestricted use, distribution, and reproduction in any medium, provided the original author and source are credited. 


\section{Cureus}

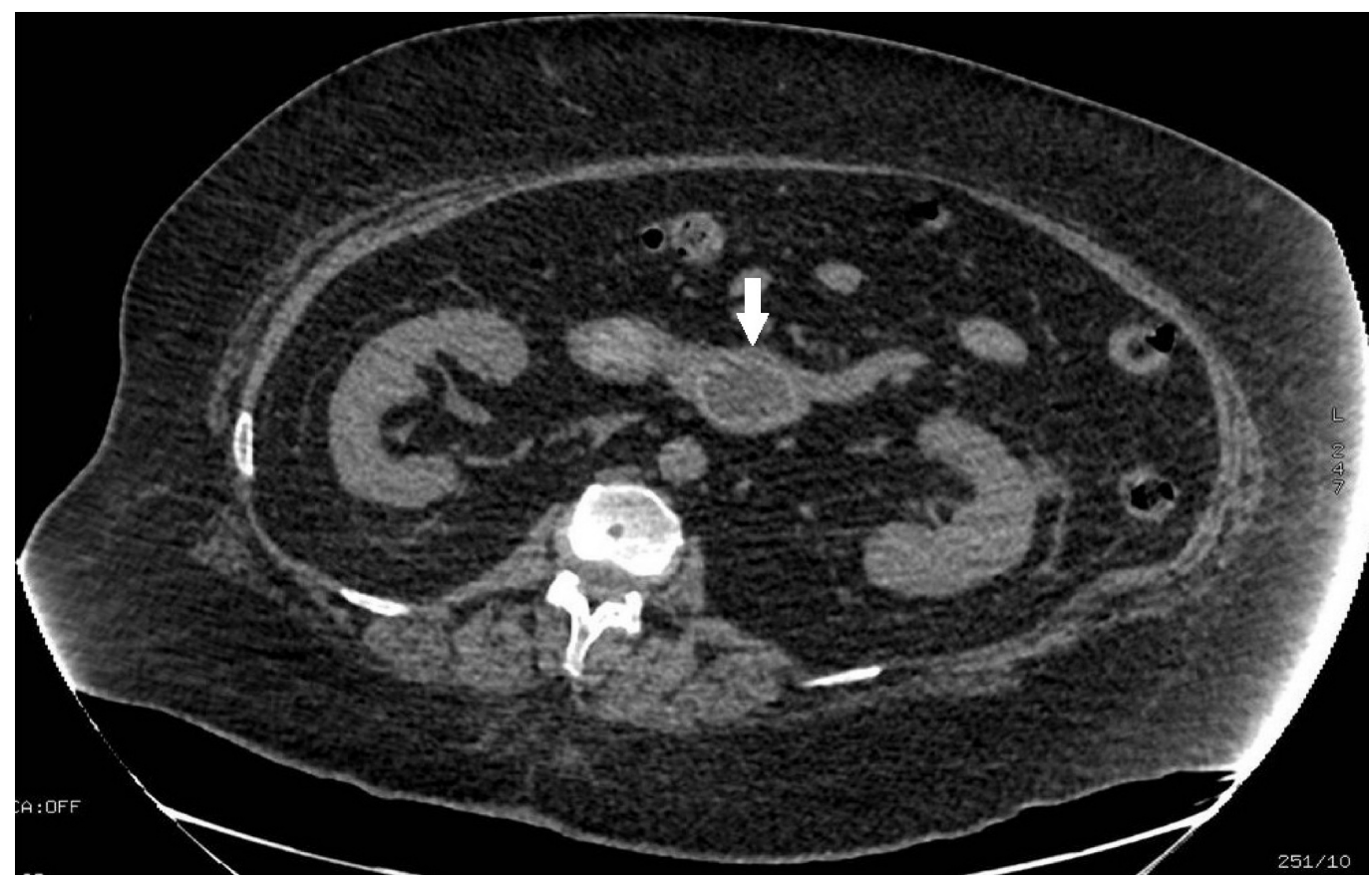

FIGURE 1: Abdominal CT scan axial cut showing the obstructing intraluminal stone in the duodenum (arrow)

CT: computed tomography

An initial improvement was noted later, followed by the recurrence of symptoms two days later. A repeat abdominal CT scan showed the migration of the duodenal stone and impaction at the distal jejunum with the dilation of the proximal loops, suggesting gallstone ileus (Figure 2).

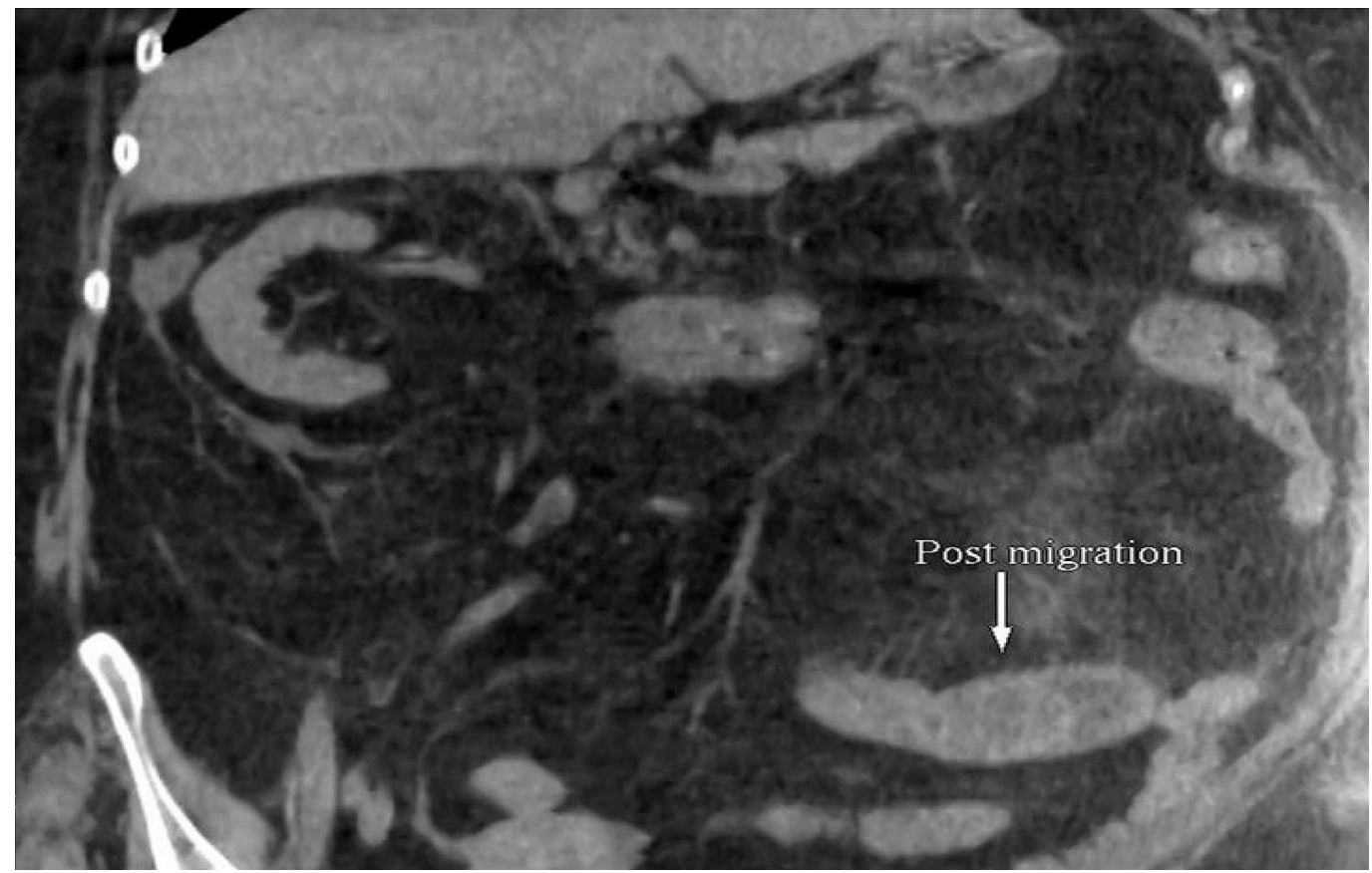

FIGURE 2: Abdominopelvic CT scan coronal cut showing the 


\section{Cureus}

\section{migration of the obstructing stone to the jejunum (arrow)}

CT: computed tomography

Subsequent jejunotomy and the successful extraction of the stone (Figure 3) were performed, with no postoperative complications.

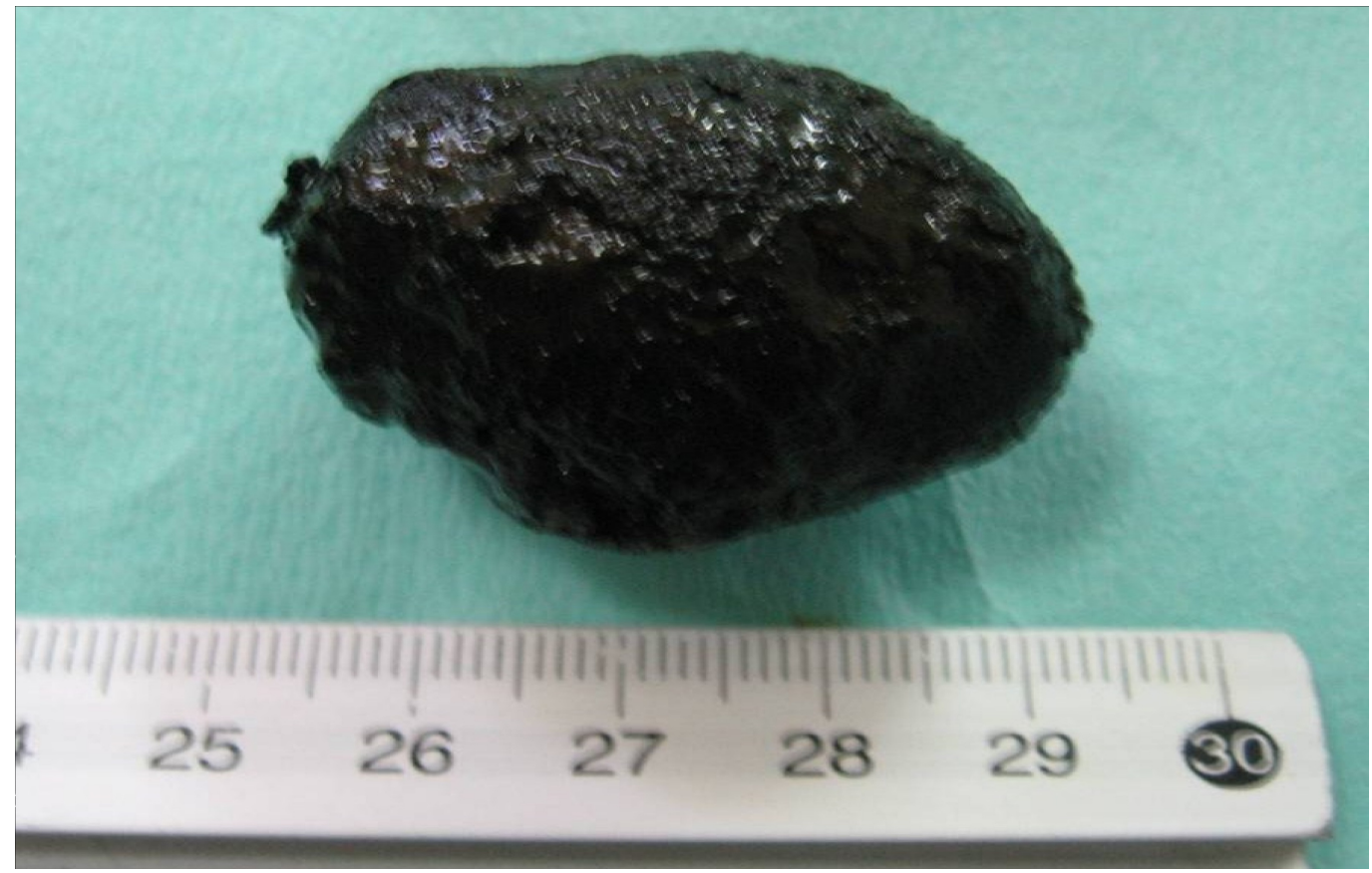

FIGURE 3: Extracted $4 \mathrm{~cm}$ gallstone following jejunotomy

\section{Discussion}

Although over 300 cases of Bouveret's syndrome have been reported, it is unusual to see a migrating gallstone after seven days of obstruction. The rarity of stone migration and its timing after the ingestion of the water-soluble contrast raised the speculation of possible "iatrogenic" migration of the stone. Conventional hyperosmolar water-soluble contrast media have pharmacologic effects, including cholinesterase inhibition, the release of histamine, and in the gut, the release of a serotonin-like substance, which enhances bowel motility [2-4].

\section{Conclusions}

We believe that the hyperosmolar contrast agent played a role in inducing osmotic pressure, intestinal smooth muscle contraction, and decreasing wall edema, which led to the spontaneous disimpaction and migration of the stone forwards. We believe this presentation is unique and warrants a heightened clinical awareness in order to avoid delayed diagnosis and management.

\section{Additional Information}

\section{Disclosures}

Human subjects: Consent was obtained by all participants in this study. Conflicts of interest: 
In compliance with the ICMJE uniform disclosure form, all authors declare the following:

Payment/services info: All authors have declared that no financial support was received from any organization for the submitted work. Financial relationships: All authors have declared that they have no financial relationships at present or within the previous three years with any organizations that might have an interest in the submitted work. Other relationships: All authors have declared that there are no other relationships or activities that could appear to have influenced the submitted work.

\section{References}

1. Reinhardt SW, Jin LX, Pitt SC, Earl TM, Chapman WC, Doyle MB: Bouveret's syndrome complicated by classic gallstone ileus: progression of disease or iatrogenic?. J Gastrointest Surg. 2013, $17: 2020-2024$. 10.1007/s11605-013-2301-7

2. Cappell MS, Davis M: Characterization of Bouveret's syndrome: a comprehensive review of 128 cases. Am J Gastroenterol. 2006, 101:2139-2146. 10.1111/j.1572-0241.2006.00645.x

3. Ceresoli M, Coccolini F, Catena F, Montori G, Di Saverio S, Sartelli M, Ansaloni L: Watersoluble contrast agent in adhesive small bowel obstruction: a systematic review and metaanalysis of diagnostic and therapeutic value. Br J Surg. 2007, 94:404-411. 10.1016/j.amjsurg.2015.06.012

4. Haddad FG, Mansour W, Deeb L: Bouveret's syndrome: literature review. Cureus. 2018, 10:e2299. doi:10.7759/cureus.2299 